le portiQue Le Portique

Revue de philosophie et de sciences humaines

13-14| 2004

Foucault : usages et actualités

\title{
Nager entre deux mots
}

À propos de « Désir et plaisir » de Gilles Deleuze

\section{Benoît Goetz}

\section{(2) OpenEdition}

Journals

Édition électronique

URL : http://journals.openedition.org/leportique/634

DOI : $10.4000 /$ leportique.634

ISSN : $1777-5280$

Éditeur

Association "Les Amis du Portique"

Édition imprimée

Date de publication : 1 septembre 2004

ISSN : 1283-8594

Référence électronique

Benoît Goetz, « Nager entre deux mots », Le Portique [En ligne], 13-14 | 2004, mis en ligne le 15 juin 2007, consulté le 26 mars 2021. URL : http://journals.openedition.org/leportique/634 ; DOI : https:// doi.org/10.4000/leportique.634

Ce document a été généré automatiquement le 26 mars 2021.

Tous droits réservés 


\title{
Nager entre deux mots
}

\author{
À propos de « Désir et plaisir » de Gilles Deleuze
}

\section{Benoît Goetz}

1 «Philosophe malgré lui » ${ }^{1}$, « Hors ou dans la philosophie » ${ }^{2}$..., la question de l'identité philosophique de Michel Foucault continue, semble-t-il, à inquiéter beaucoup. Cela n'a jamais été le problème de Gilles Deleuze qui commence son intervention, "Qu'est-ce qu'un dispositif? », par la formule : « La philosophie de Foucault... » ${ }^{3}$.

2 Pour Deleuze, cette question n'a pas même à être posée. L'intérêt de la rencontre Deleuze-Foucault est donc, à mon sens, d'abord celle-ci : d'éclairer en pleine lumière ou d'extraire la philosophie d'un penseur qui n'a jamais voulu ou désiré l'exposer en tant que telle. Non par mépris pour la philosophie mais par refus de l'identification ${ }^{4}$. Il faut s'avancer masqué, tel un "philosophe masqué " ${ }^{5}$, non par prudence ou coquetterie, mais pour laisser se faire le travail de transformation de la pensée qui ne laisse aucune identité intacte. Les vocables comme " philosophe ", " philosophie ", et même, mais à un moindre degré (pourquoi ?) "pensée » sont des poids et des maux. Le nom propre lui-même, son propre nom, peut devenir un écran qu'il faut savoir brouiller à l'occasion. Au lecteur alors de se débrouiller, c'est-à-dire de travailler pour aller, audelà des panneaux, vers ce qui introduit « une différence significative dans le champ du savoir ». Cette lutte avec les vocables, que d'autres ont nommée " écriture », est un jeu de vérité où se confondent la peine et le plaisir ${ }^{6}$.

3 Mais voilà : "plaisir » est un autre vocable dont Foucault a fait le choix, en le pluralisant et en l'«hellénisant» (les aphrodisia) pour, comme le note justement François Wahl, se déprendre du désir, « de “notre" conceptualité du désir » ${ }^{7}$. Or, tel n'a pas été le choix de Gilles Deleuze qui, dans la voie ouverte par Spinoza, a tenté de nous déprendre de la conceptualité traditionnelle du désir-manque par l'affirmation du Désir lui-même - qui ne manque de rien et surtout pas des "plaisirs » qui seraient plutôt pour lui des embûches qui le mèneraient à sa perte.

4 Ce qui est du plus haut intérêt dans cette querelle concernant le choix d'un mot, c'est qu'il s'agit, pour Deleuze comme pour Foucault, de fabriquer une machine de guerre pour lutter contre le même mal : un « dispositif de sexualité » qui arraisonne le corps occidental. Depuis quand et comment? C'est cette question que posent ensemble 
Deleuze et Foucault. Le corps-organisme, plié, ou plutôt replié et écrasé par son organisation totalitaire, le corps vissé sur une sexualité obligatoire qui a été proclamée sa « vérité », tel est leur ennemi commun.

Deleuze n'est donc pas seulement « lecteur », qui plus est avisé, de Foucault. Sa pensée entre en résonance avec la sienne. Déclarer de but en blanc Foucault philosophe ne ressemble donc en rien à une procédure d'appropriation disciplinaire (du genre : « moi, Gilles Deleuze, philosophe incontesté, certifie que...» Horreur!), non, Deleuze aide le lecteur au travail à comprendre que l'usage des plaisirs et le Désir qui tend le corps sans organe sont des contre-attaques menées par des machines de guerre alliées. Question de choix des mots pour lutter contre les mêmes maux.

Il faut comprendre que le "plaisir » de Foucault ainsi que le «désir» de Deleuze ne sont qu'homonymes des vocables qu'ils contredisent. Tel est le jeu de vérité extraordinaire auquel il nous est donné d'assister dans la «lettre » écrite par Gilles à Michel publiée par le Magazine littéraire sous le titre "Désir et plaisir " ${ }^{8}$. Dans cette série de notes que François Ewald devait remettre à Foucault après la publication de la Volonté de savoir, Deleuze demande : « Est-ce que je pourrais penser à des équivalence du type : ce qui pour moi est "corps sans organes-désirs" correspond à ce qui, pour Michel est "corps-plaisirs"?"

7 Si Foucault, comme il le confie à Deleuze, ne peut pas supporter le mot « désir ", c'est parce qu'il ne parvient pas à le dissocier du manque, fidèle, en ce sens, à une tradition qui va de Platon jusqu'à Sartre, en passant, bien sûr, par la psychanalyse. Or si Deleuze ne supporte pas lui le mot "plaisir", c'est parce qu'il lui semble que le plaisir interrompt « le processus immanent au désir " ${ }^{9}$. Pour Deleuze, « c'est de la même façon que le désir est rapporté à la loi du manque et à la norme du plaisir» ${ }^{10}$. La question n'est donc pas de savoir quelle interprétation du désir est la bonne. En fait, Foucault, comme Deleuze, ne veut pas du désir-manque, complice du dispositif de sexualité dont il cherche à nous déprendre ${ }^{11}$.

8 Quant à Deleuze, il entrevoit très bien que les plaisirs de Foucault, les aphrodisia, «arriment des contre-pouvoirs », même s'il ne sait pas très bien encore de quelles manières (en 1977, Foucault n'avait pas encore publié l'Usage des plaisirs). En fait, Foucault pas plus que Deleuze ne croit en la vertu intrinsèque de plaisir. Il s'intéresse aux modes de subjectivation qui procèdent de certains usages des plaisirs. Les aphrodisia ne sont ni moyens ni fins. Ils sont des expériences où le sujet se constitue et se déconstitue. Ils s'insèrent dans ces «jeux de vérité » qui mêlent problématisations et pratiques, où le sujet comme tel se produit. La vérité se joue à travers des pratiques qui sont elles-mêmes des formes de problématisation et de subjectivation. C'est bien pourquoi les aphrodisia ne sont pas ces miroirs aux alouettes qui mobiliseraient les sujets d'un sexe conçu éternellement comme désir-manque, soumis, de plus, au phallus, à la loi et à la castration. Les aphrodisia, et leur usage, sont la condition de possibilité d'un certain dégagement hors des normes du sexe. Cela a pu avoir lieu en Grèce, donc... On comprendra alors, avec Deleuze que, si Foucault est philosophe, c'est parce que son histoire de la sexualité est inséparable d'une histoire de la vérité qui n'a rien à voir avec une histoire des pratiques sexuelles ni avec une histoire des doctrines sur l'être.

9 Il faut finalement faire l'hypothèse d'une ontologie cachée de Foucault que Deleuze aura mise à découvert. On la trouve condensée dans cette page qui expose «le diagramme de Foucault » et qui accompagne un de ces extraordinaires schémas dont Deleuze a le secret (et que nous reproduisons ici). 


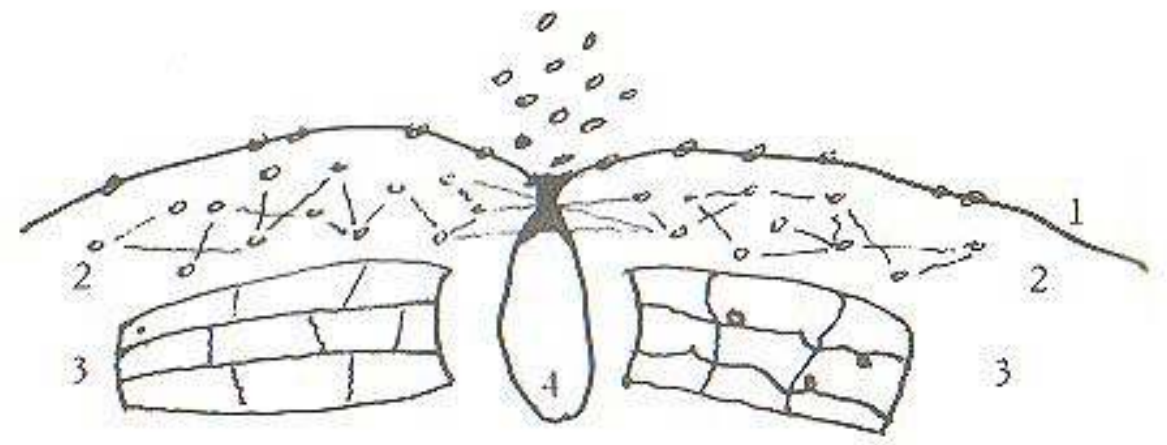

1 : ligne du dehors

$2:$ zone stratégique

3 : strates

4 : pli (zone de subjectivation).

10 «Le monde est fait de surfaces superposées, archives ou strates. Aussi le monde est-il savoir. Mais les strates sont traversées d'une fissure centrale, qui répartit d'un côté les tableaux visuels, de l'autre côté les courbes sonores: l'énonçable et le visible sur chaque strate, les deux formes irréductibles du savoir, Lumière et Langage, deux vastes milieux d'extériorité où se déposent respectivement les visibilités et les énoncés. Alors nous sommes pris dans un double renversement. Nous nous enfonçons de strate en strate, de bandelettes en bandelettes, nous traversons les surfaces, les tableaux et les courbes, nous suivons la fissure, pour essayer d'atteindre à un intérieur du monde : comme dit Melville, nous cherchons une chambre centrale, avec la peur qu'il n'y ait personne, et que l'âme de l'homme ne révèle un vide immense et terrifiant (qui songerait à chercher la vie dans les archives ?). Mais en même temps, nous essayons de monter au-dessus des strates, pour atteindre à un dehors, à un élément atmosphérique, à une "substance non stratifiée" qui serait capable d'expliquer comment les deux formes du savoir peuvent s'étreindre et s'entrelacer sur chaque strate, d'un bord à l'autre de la fissure... ${ }^{12}$

11 Avant d'inventer une philosophie nouvelle (à partir de Différence et répétition, 1969), Deleuze avait fait le portrait de grands philosophes de la tradition: Hume (1953), Nietzsche (1962), Kant (1963), Bergson (1966). En 1986, il retrouve cette activité de portraitiste pour extraire de l'œuvre de son ami une "image de la pensée » qui entretient avec la sienne de très remarquables rapports. Cela pourrait être l'amitié de deux philosophes: non pas la rivalité dans la recherche de la vérité, mais une complicité telle qu'à certains moments, des pensées éloignées entrent en résonance. On ne parlera plus alors de lecture ni d'interprétation. Un perspectivisme plus radical conduit à considérer le «Foucault de Deleuze » comme un portrait d'autant plus exact qu'il est totalement reconstruit à travers le dispositif conceptuel deleuzien. Le signe en est que dans « Désir et plaisir » est sans cesse évoquée une philosophie foucaldienne qui n'est pas encore développée (et que, bien sûr, Foucault n'aurait jamais produit comme telle). Ainsi : "Michel, je crois, n'a pas encore développé ce point: sa conception originale des rapports de force, ce qu'il appelle rapport de force, et qui doit être un concept aussi nouveau que tout le reste. ${ }^{13}$

12 Dans ce texte de 1977, Deleuze attend que Foucault dise du nouveau sur des concepts qu'il a déjà totalement recréés : rapports de forces, vérités, plaisirs. C'est cette attente qui explique la prodigieuse tension de ce texte ${ }^{14}$. Pourtant il est très frappant que, dans 
"Désir et plaisir", Deleuze ne pose aucune question à son ami. Ces notes de travail étaient destinées à être transmises à Foucault. Mais Deleuze ne formule que des questions qu'il se pose, à lui-même. On ne trouvera évidemment aucune objection, ni l'ébauche de la moindre controverse.

Amitié d'astre ${ }^{15}$ : « Désir et plaisir » est pour nous, aujourd'hui, un texte extraordinaire en ce qu'il maintient vivante, et, pour ainsi dire à l'état brut, une amitié philosophique au travail. C'est aussi le genre de la lettre philosophique, et toute une conception de la communication entre philosophes, qui se trouvent là bouleversés et renouvelés.

\section{NOTES}

1.. John RAJCHMAN, « Foucault : l'éthique et l'œuvre », Michel Foucault philosophe, rencontre internationale, Paris 9-11 janvier 1988, «Des travaux », Seuil, 1989, p. 249.

2.. François WAHL, « Hors ou dans la philosophie ", Michel Foucault philosophe, op. cit., p. 85.

3.. Gilles DELEUZE, «Qu'est ce qu'un dispositif? », Michel Foucault philosophe, op. cit., p. 185.

4.. Jacques Rancière fait à ce propos des remarques pertinentes : « Michel Foucault parlait un jour d'une philosophie qui serait un peu comme une guérilla, où le philosophe ne serait jamais là où on l'attendait. L'intérêt n'est évidemment pas de se déplacer pour être insaisissable. Ne pas parler de soi, raconter des histoires de fous ou d'ouvriers au lieu d'énoncer des thèses sur l'Être et l'Un, ce n'est sûrement pas une manière d'assurer l'identité à soi du malin qui se dérobe aux contrôles public d'identité. C'est consentir à une autre forme de cette altération, de ce devenir-autre qui affecte aussi bien celui qui parle de lui et celui qui n'en parle pas. Quelle que soit la voie choisie pour penser, on n'échappe pas à cette nécessité énoncée en des langages très différents par le romancier - Flaubert ou Proust - et par le philosophe Deleuze, la nécessité de devenir en même temps quelque chose qui ne pense pas. » Jacques RANCIÈRE, « La philosophie en déplacement », La Vocation philosophique, Paris, Bayard, 2004, p. 33-34. 5.. Michel Foucault, « Le philosophe masqué » (1980), Dits et écrits, T. IV, p.104-110. Voir également, Jean zounGRANA, « Le philosophe masqué », Le Portique n 7, 2001, p. 177-189. 6.. Voir la définition du travail proposée par Michel Foucault, Jean Claude Milner, Paul Veyne et François Wahl, pour leur collection « Des travaux » au Seuil : « Travail : ce qui est susceptible d'introduire une différence significative dans le champ du savoir, au prix d'une certaine peine pour l'auteur et le lecteur, et avec l'éventuelle récompense d'un certain plaisir, c'est-à-dire d'un accès à une autre figure de la vérité ".

7.. François WAHL, op. cit., p. 94.

8.. « Désir et plaisir », Magazine Littéraire n 325, octobre 1994. Repris in G. DELEUZE, Deux régimes des fous, Paris, Éditions de Minuit, 2003.

9.. Ibid., p. 64.

10.. Ibid. 
11.. Du lecteur de Deleuze et Foucault on pourrait alors dire, pour parodier ce dernier, qu'il est un « nageur entre deux mots ». Voir « André Breton, un nageur entre deux mots ", Dits et écrits, T. I, p. 554-557.

12.. Gilles DELEUZE, Foucault, Paris, Éditions de Minuit, 1986, p. 128-129.

13.. « Désir et plaisir », op. cit., p. 60.

14.. En 1986, il développera lui-même cette nouvelle conception. Voir Foucault, op. cit., p. 131: « le principe général de Foucault est : toute forme est un composé de rapports de forces... ". Le livre de 1986 se présentera lui comme un monument, au sens précis et nouveau que Deleuze donne à ce terme et qui n'a rien de commémoratif (voir Qu'est ce que la philosophie?).

15.. L'expression est de Nietzsche dans le Gai savoir.

\section{RÉSUMÉS}

Il s'agit ici de quelques remarques à propos d'un aspect de la « lecture » de Foucault par Deleuze tel qu'il se dégage d'un texte de ce dernier intitulé « Désir et plaisir».

These are a few notes about one aspect of Deleuze's reading of Foucault as we may see it emerge from "Desire and Pleasure", a text written by Deleuze. 